\title{
Adenine Inhibits B16-F10 Melanoma Cell Proliferation
}

\author{
Prashanta Silwal ${ }^{*}$ and Seung-Kiel Park ${ }^{\dagger, * *}$ \\ Department of Biochemistry, College of Medicine, Chungnam National University, Daejeon 35015, Korea
}

\begin{abstract}
Adenine, a purine base, is a structural component of essential biomolecules such as nucleic acids and adenine nucleotides. Its physiological roles have been uncovered. Adenine suppresses IgE-mediated allergy and LPS-induced inflammation. Although adenine is known to inhibit lymphocyte proliferation, the effect of adenine to melamoma cells is not reported. Here, we investigated the growth inhibitory effects of adenine on B16-F10 mouse melanoma cells. Adenine suppressed the proliferation of B16-F10 cells in dose-dependent manner with the maximal inhibitory dose of $2 \mathrm{mM}$. Adenine treatment induced cell death molecular markers such as PARP and caspase 3 cleavages. Pan-caspase inhibitor z-VAD dramatically rescued the cell death molecular markers, cell proliferation recovered marginally. These results provide the possibility of adenine to be used as an anti-tumor agent.
\end{abstract}

Key Words: Adenine, Melanoma, Cell death, Tumor, Apoptosis

\section{INTRODUCTION}

The purine adenine is a component of biomolecules, such as nucleic acids and adenine nucleotides. It is produced mainly from 5'-methylthioadenosine (MTA) by MTAphosphorylase (Avila et al., 2004). Adenine production from the MTA degradation contributes $85 \sim 97 \%$ of total adenine synthesis in dividing human lymphoblastoid cells (Kamatani and Carson, 1981). Adenine promotes survival of Purkinje cells (Watanabe et al., 2003) and erythrocytes in whole blood storage (Simon et al., 1962). In contrast to its survival effects, adenine inhibits the growth of lymphoblast (Hershfield et al., 1977; Snyder et al., 1978) independent of adenine salvage enzyme APRT (Hershfield et al., 1977). In our previous report we showed that adenine inhibits IgE-mediated mast cell activation and allergic reactions in vitro and in vivo (Silwal et al., 2015). Adenine also inhibits the immune function of human mononuclear leukocytes (Kishi et al., 1985). Adenine is cell-permeable and convertible to adenosine monophosphate (AMP) via adenine salvage pathway. AMP and ATP pools are replenished during the salvage thereby contributing to its cellular and physiological activities. AMP formation from adenine contributes to activation of AMPK in various cells (Young et al., 2015).

The programmed cell death, or apoptosis, is an orderly and genetically controlled form of cell demise. It is a coordinated and usually energy-dependent process which involves the activation of a group of cysteine aspartyl proteases, caspases (Elmore, 2007). Caspase-3 is an execution caspase in apoptosis that can be activated by external or intrinsic stimuli. The Bcl-2 (B-cell lymphoma-2) family proteins, including both anti apoptotic (Bcl-2, Bcl-XL, and Mcl-1) and proapoptotic (Bid, Bax, and Bad) members are particularly

Received: May 29, 2020 / Revised: July 23, 2020 / Accepted: July 24, 2020

* Graduate Student, ${ }^{* *}$ Professor.

${ }^{\dagger}$ Corresponding author: Seung-Kiel Park. Department of Biochemistry, College of Medicine, Chungnam National University, 266 Munhwa-ro, Jung-gu, Daejeon 35015, Korea.

Tel: +82-42-580-8224, Fax:+82-42-580-8121, e-mail: parksk@cnu.ac.kr

(C) The Korean Society for Biomedical Laboratory Sciences. All rights reserved.

(c) This is an Open Access article distributed under the terms of the Creative Commons Attribution Non-Commercial License (http://creativecommons.org/licenses/by-nc/3.0/) which permits unrestricted non-commercial use, distribution, and reproduction in any medium, provided the original work is properly cited. 
important in the caspase activation (Cory and Adams, 2002). AMP-activated protein kinase (AMPK) participates in the development and progression of tumors by regulating cellular energy. AMPK is a metabolic checkpoint regulating energy homeostasis, and several AMPK activators are being used in cancer therapy because of its involvement in inhibiting tumor cell growth and inducing apoptosis (Theodoropoulou et al., 2013). AMP activates AMPK by inhibiting dephosphorylation of phosphorylated AMPK as well as acts as an allosteric activator of AMPK (Gowans et al., 2013).

Previous studies showed that adenine is cytotoxic to lymphoblast (Hershfield et al., 1977; Snyder et al., 1978) and has anti-tumor activity for colon (Han et al., 2017), cervical (Lai et al., 2019) and hepatic (Su et al., 2020) cancer cells. However, its effect on melanoma cells is yet to be studied. Melanoma is the most aggressive form of skin cancer which is highly metastatic with the major problem being its pronounced resistance to therapy (Soengas and Lowe, 2003). Cancer therapies target the elimination of tumor cells through apoptotic cell death. The ineffectiveness of chemotherapy in treating melanoma is mainly due to improper functioning of the apoptosis programs. Therefore, the therapeutic approaches that either alone or in combination induce apoptosis are crucial for the proper treatment of melanoma (Lowe and Lin, 2000).

In this study, we investigated the adenine induced cell death on B16-F10 murine melanoma cells. We show that adenine elicits growth inhibition and apoptotic cell death by activation of caspase in B16-F10 melanoma cells.

\section{MATERIALS AND METHODS}

\section{Materials}

The following materials were purchased from the indicated commercial sources: adenine, thiazolyl blue tetrazolium bromide (MTT), z-VAD-fmk and dimethyl sulfoxide (DMSO) from Sigma-Aldrich (St Louis, MO, USA); fetal bovine serum (FBS) from Gibco/Life Technologies (Grand Island, NY, USA); Cytotoxicity Detection Kit plus (LDH) from Roche Diagnostic GmBH (Mannheim, Germany); Dulbecco's Modified Eagle Medium (DMEM) from Welgene (South Korea); anti-cleaved caspase-3 antibodies from Cell Sig- naling Technology (Beverly, MA, USA); anti-PARP from Santa Cruz (CA, USA); RIPA lysis buffer from ELPIS Biotech (South Korea); ECL chemiluminescence kit from Millipore (Billerica, MA,USA); B16-F10 melanoma cell line from American Type Culture Collection.

\section{Cell culture}

B16-F10 melanoma cells were cultured in DMEM supplemented with $10 \%$ FBS and standard concentration of penicillin/streptomycin antibiotics. Cultures were maintained in a standard condition in $5 \% \mathrm{CO}_{2}$ incubator at $37^{\circ} \mathrm{C}$. For all experiments, required numbers of cell were incubated for $24 \mathrm{~h}$ in appropriate plates. Then various doses of adenine or other compounds were treated for $24 \mathrm{~h}$ in a complete growth medium and cells were harvested according to required experimental purposes.

\section{Cell viability assay}

Cell viability was determined using thiazolyl blue tetrazolium bromide (MTT) and cell counting. For MTT assay, cells were seeded into 96 -well plates $\left(1 \times 10^{3}\right.$ cells/well $)$ and incubated for $24 \mathrm{~h}$ at $37^{\circ} \mathrm{C}$. The cells were then treated with various doses of adenine for another $24 \mathrm{~h}$ which were then incubated with MTT $(5 \mathrm{mg} / \mathrm{mL})$ for $2 \mathrm{~h}$ and the formazan produced was dissolved in DMSO. Absorbance was measured at $570 \mathrm{~nm}$ in a microplate reader (Thermo Fisher Scientific, Waltham, MA, USA). Data are presented as percentage of untreated control group. For cell counting assay, $1 \times 10^{5}$ cells per $\mathrm{mL}$ were seeded in 6 -well plates. After incubating overnight, cells were treated with adenine and were trypsinized after indicated time. $10 \mu \mathrm{L}$ of cell were mixed with equal volume of $0.4 \%$ Trypan blue and $10 \mu \mathrm{L}$ of mixture was used in cell counting using automated cell counter.

\section{Lactate dehydrogenase (LDH) assay}

Non-radioactive colorimetric assay was performed using Cytotoxicity Detection Kit (LDH). B16-F10 cells seeded and incubated overnight in 96-well plates were treated with adenine for $24 \mathrm{~h}$. Then as per the manufacturer's protocol, catalyst and dye solution mixture (reaction mixture) was added to the cells and incubated for $30 \mathrm{~min}$ at room tem- 
perature, protecting plates from lights. Resulting values were used to calculate LDH release percentage using the following formula: LDH release (cytotoxicity) $(\%)=($ experimental value - low control)/(high control - low control) $\times 100$. To determine the effect of adenine on cell proliferation using LDH assay, B16-F10 cells were seeded in presence or absence of adenine in 96 well plates and were incubated for $24 \mathrm{~h}$.

\section{Western blot analysis}

Cells were collected, washed with ice-cold PBS, and then lysed in ice-cold lysis buffer for $30 \mathrm{~min}$ on ice. Lysates were micro-centrifuged at $12,000 \mathrm{rpm}$ at $4^{\circ} \mathrm{C}$ for $20 \mathrm{~min}$ and protein concentration was measured using Bradford assay. After SDS-PAGE, proteins in the gel were transferred to a
PVDF membrane. The membrane was blocked in 5\% skim milk in Tris-buffered saline containing $0.1 \%$ Tween 20 (TBS-T) for $1 \mathrm{~h}$ and then incubated overnight with primary antibodies at $4{ }^{\circ} \mathrm{C}$. After washing the membrane with TBS-T, immune-reactive proteins were detected with horseradish peroxidase-conjugated secondary antibody using chemiluminescence kit. Densitometry analysis was performed using ImageJ software.

\section{Statistical analysis}

Data are presented as means \pm SEM. Statistical analysis were performed by using GraphPad Prism software. The data from adenine-treated and adenine-untreated groups of cells were analyzed with Student's $t$-test and $P \leq 0.05$ was
A

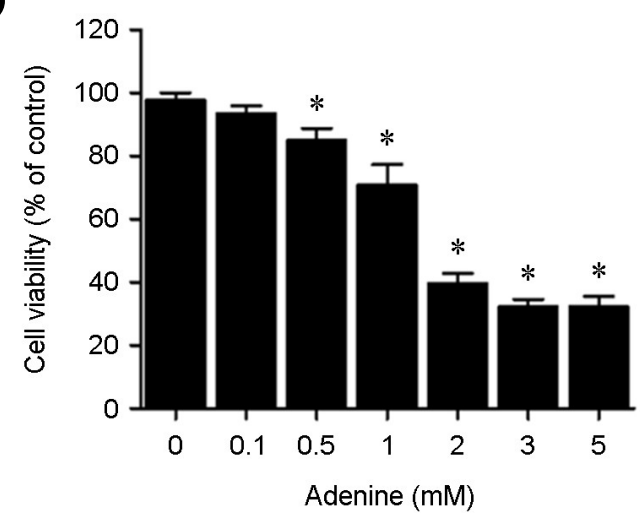

B

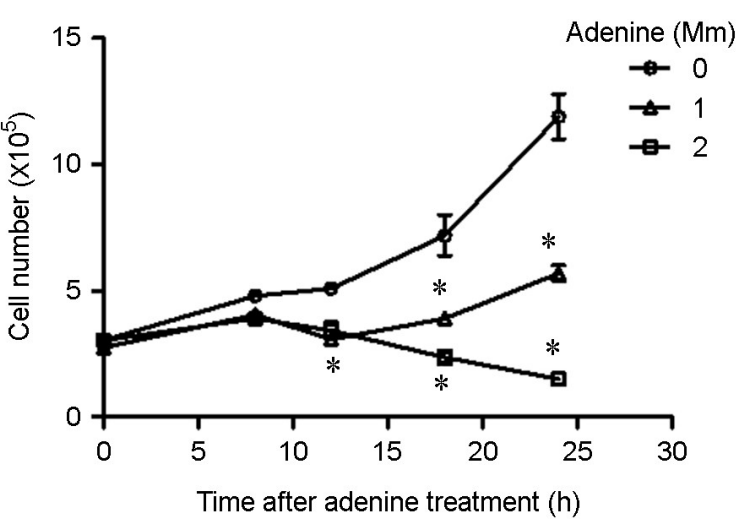

E

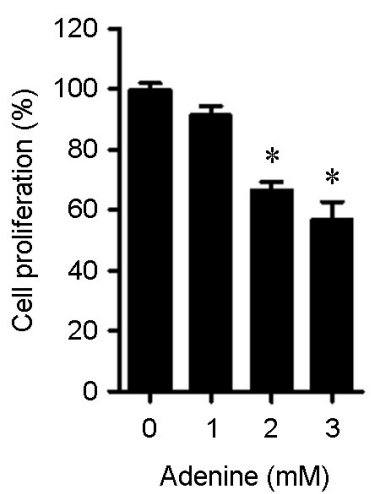

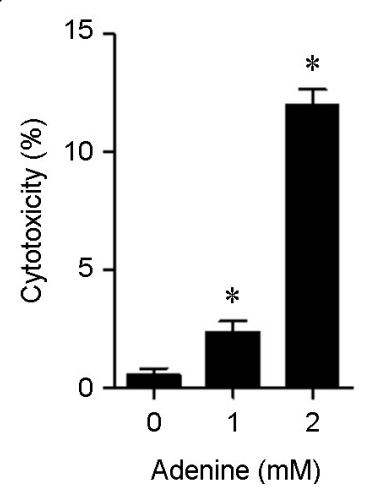

Fig. 1. Adenine inhibits cell viability and induces death of B16-F10 melanoma cells. (A) B16-F10 cells were treated with indicated doses of adenine for $24 \mathrm{~h}$ in complete media and cell viability was measured using MTT assay (A) and viable cell count (B). The MTT data are presented as percentage of non-treated control group. (C) Hypoxanthine was treated in similar condition as that of adenine and MTT assay was performed to determine cell viability after $24 \mathrm{~h}$. (D, E) B16-F10 cell treated with indicated doses of adenine for $24 \mathrm{~h}$ in complete media and LDH release was determined using LDH assay kit as described in materials and method section. Data are means \pm SEM of three independent experiments. Significant difference against control group is indicated as $* P<0.05$. 
considered to indicate significance difference.

\section{RESULTS}

Adenine inhibits the cell growth and cell viability in B16-F10 melanoma cells

The effect of adenine on growth of B16-F10 melanoma cells was evaluated by MTT assay and cell counting. Adenine treatment caused a dose-dependent decrease in cell viability as compared to untreated cells with maximal inhibition at $2 \mathrm{mM}$ dose (Fig. 1A). Adenine treatment caused reduction in cell number in time dependent manner as seen on cell counting assay (Fig. 1B). Adenine treatment had no significant effect on cell numbers till $8 \mathrm{~h}$ but significant drop in numbers were observed after $12 \mathrm{~h}$ of treatment suggesting adenine inhibits cell growth and causes cell death after $12 \mathrm{~h}$ (Fig. 1B). To know if the effect is adenine specific, a structural analog of adenine, hypoxanthine, was used. Hypoxanthine did not inhibit the B16-F10 melanoma cell viability (Fig. 1C). Lactate dehydrogenase (LDH) assay was performed to assess the cell proliferation and cytotoxicity by measuring total $\mathrm{LDH}$ and $\mathrm{LDH}$ released in medium after adenine treatment respectively. Adenine treated at the time of cell seeding and measuring the LDH after $24 \mathrm{~h}$ showed inhibited level of LDH confirming inhibition of cell proliferation by adenine (Fig. 1D). Adenine caused excessive release of LDH which suggest that it is cytotoxic as it affects the membrane integrity of the B16-F10 cells (Fig. 1E). These results revealed that adenine decreases proliferation and kill the B16-F10 melanoma cells.

\section{Adenine induces apoptosis in B16-F10 melanoma cells}

To confirm if the cell death induced by adenine in apoptotic cell death, Western blotting was performed to see molecular cleavages of caspase- 3 and PARP proteins. We measured cleavage formation of caspase-3, an executor caspase that is activated through both intrinsic and extrinsic apoptosis pathways (Taylor et al., 2008). As shown, expression of cleaved form of caspase- 3 was induced by adenine treatment (Fig. 2A). In addition, the activation of caspase-3 leads to the cleavage of several proteins, one of which is PARP. Although PARP is not essential for cell death, the cleavage
(A)

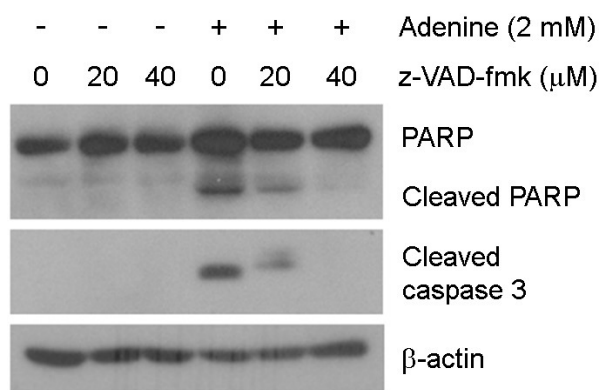

B

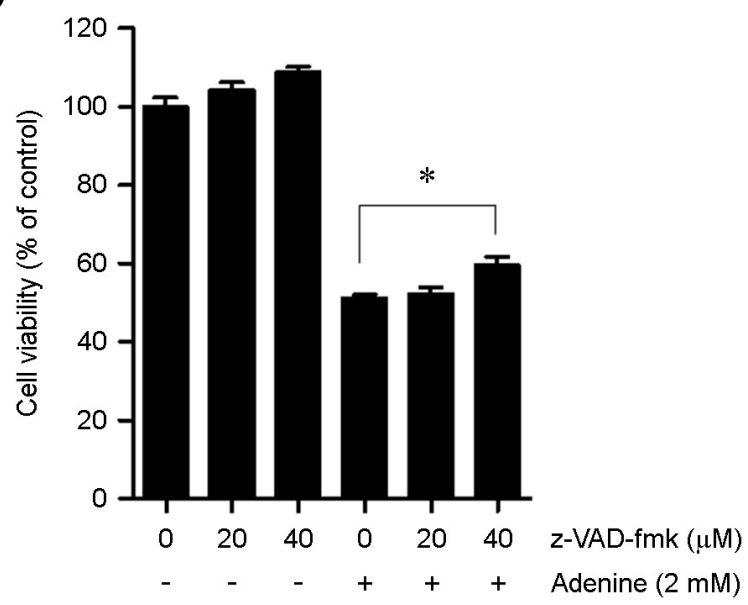

C

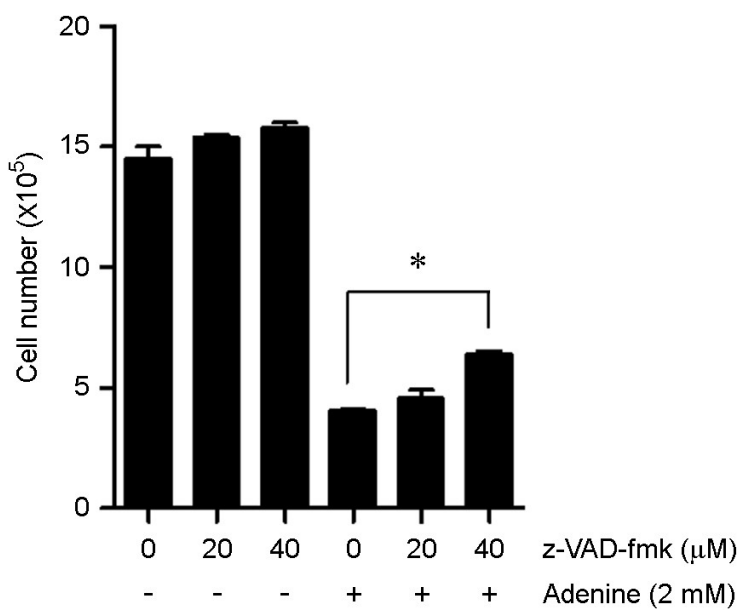

Fig. 2. Adenine induces apoptosis of B16-F10 melanoma cells. (A) B16-F10 cells were pre-incubated with pan-caspase inhibitor $\mathrm{z}$-VAD-fmk for $1 \mathrm{~h}$ and exposed to adenine for $24 \mathrm{~h}$. Cell lysates were used for Western blotting to detect indicated proteins. (B) MTT assay and (C) Cell count assay were performed with cells pre-incubated and treated with z-VAD and adenine as described above. Data are means \pm SEM of three independent experiments Significant difference against control group is indicated as $* P<0.05$. 
of PARP is another hallmark of apoptosis (Kaufmann et al., 1993). Adenine increased the expression of cleaved PARP (Fig. 2A).

Cleavage form of caspase- 3 suggests that adenine induces the caspase-dependent apoptosis in B16-F10 cells. To confirm this, we used a pan-caspase inhibitor z-VAD and results shows that it reduced the adenine induced cleaved-PARP and -caspase-3 (Fig. 2A). Cell viability analysis with MTT assay (Fig. 2B) and viable cell count data (Fig. 2C) showed z-VAD $(40 \mu \mathrm{M})$ significantly rescued B16-F10 cell death resulted from adenine treatment. These results suggested that adenine induces caspase-dependent apoptosis in B16F10 cells.

\section{DISCUSSION}

We showed in this study that adenine induces cell death of B16-F10 melanoma cells by activating caspase-3. We demonstrated that adenine reduced the cell viability and caused apoptotic cell death. Adenine inhibited cell growth as measured by MTT assay (Fig. 1A) and viable cell counting (Fig. 1B). Also, adenine induced the cleavage of PARP in B16-F10 cells with a dose of $2 \mathrm{mM}$ or more (Fig. 2A). Proteolytic cleavage of PARP is a marker of apoptosis. Apoptosis is mediated by proteases called caspases (Kerr et al., 1972; Taylor et al., 2008). Caspase-3 is primarily responsible for the cleavage of PARP during cell death (Thornberry, 1997). In the present study, we found that adenine treatment increased the caspase-3 cleavage (Fig. 2A). Blocking apoptosis with pan-caspase inhibitor z-VAD partially protected B16-F10 cells from adenine induced cell death (Fig. 2). These results suggested that adenine killed the melanoma cell death partly by inducing caspase-dependent apoptosis.

Adenine is cell-permeable and can be converted to AMP in cells by an enzyme APRT (Kamatani and Carson, 1981). AMPK activation leading to apoptosis may be cell-type dependent (Zadra et al., 2014). The AMP thereby activates AMPK to promote various physiological effects. Several reports suggest that adenine could be a novel and simplest AMPK activator (Cheng et al., 2015). AMPK activation has also been linked with induction of cell cycle arrest and cell death (Vincent et al., 2015). Anti-tumor effects of adenine for colon (Han et al., 2017), cervical (Lai et al., 2019) and hepatic ( $\mathrm{Su}$ et al., 2020) cancer cells are mediated from adenine-induced cell cycle arrest and AMPK activated. Therefore, the cytotoxic effect of adenine on melanoma cells shown here may be through AMPK activation of adenine in melanoma cells.

Another mechanism of adenine induced cytotoxicity could be transmethylation as in W1-L2 cells (Kishi, 1985), adenine inhibits S-adenosylhomocysteine hydrolase and causes accumulation of S-adenosylhomocysteine and growth inhibition where S-adenosylhomocysteine is a well-known transmethylases inhibitor.

Adenine showed cytotoxicity in B16-F10 melanoma cells (Fig. 1). Although its cytotoxic mechanism was inferred that excess production of adenine nucleotides through adenine salvage pathway makes an imbalance on the ratio of purine /pyrimidine nucleotides, the cytotoxicity is independent of the salvage pathway (Hershfield et al., 1977; Snyder et al., 1978). In contrast, adenine protects rat Purkinje cells in rat cerebellar primary cultures and erythrocytes in the whole blood preservation (Watanabe et al., 2003; Simon et al., 1962). Also, patients deficient in adenine phosphoribosyltransferase (APRT) and knock-out mice of the gene are viable (Engle et al., 1996). Therefore, the cytotoxicity of adenine could depend on cell types, cellular environments and the concentration of adenine.

In conclusion, this study is the first to report that adenine inhibits cell growth in B16-F10 melanoma cells partly by inducing apoptotic cell death. This study can provide evidence that adenine could be a valuable resource for the development of new agents for cancer treatment including melanoma.

\section{ACKNOWLEDGEMENT}

This work was financially supported by research funds of Chungnam National University.

\section{CONFLICT OF INTEREST}

The authors have no conflict of interest with regards to this study. 


\section{REFERENCES}

Avila MA, Garcia-Trevijano ER, Lu SC, Corrales FJ, Mato JM. Methylthioadenosine. Int J Biochem Cell Biol. 2004. 36: 2125-2130.

Cheng YF, Young GH, Lin JT, Jang HH, Chen CC, Nong JY, Chen PK, Kuo CY, Kao SH, Liang YJ, Chen HM. Activation of AMP-Activated Protein Kinase by Adenine Alleviates TNF-Alpha-Induced Inflammation in Human Umbilical Vein Endothelial Cells. PLoS One. 2015.10: e0142283.

Cory S, Adams JM. The Bcl2 family: regulators of the cellular life-or-death switch. Nat Rev Cancer. 2002, 2: 647-656.

Elmore S. Apoptosis: a review of programmed cell death. Toxicol Pathol. 2007. 35: 495-516.

Engle SJ, Stockelman MG, Chen J, Boivin G, Yum MN, Davies PM, Ying MY, Sahota A, Simmonds HA, Stambrook PJ, Tischfield JA. Adenine phosphoribosyltransferase-deficient mice develop 2,8-dihydroxyadenine nephrolithiasis. Proc Natl Acad Sci U S A. 1996. 93: 5307-5312.

Gowans GJ, Hawley SA, Ross FA, Hardie DG. AMP is a true physiological regulator of AMP-activated protein kinase by both allosteric activation and enhancing net phosphorylation. Cell Metab. 2013.18: 556-566.

Han M, Cheng X, Gao Z, Zhao R, Zhang S. Inhibition of tumor cell growth by adenine is mediated by apoptosis induction and cell cycle S phase arrest. Oncotarget. 2017. 8: 94286-94296.

Hershfield MS, Snyder FF, Seegmiller JE. Adenine and adenosine are toxic to human lymphoblast mutants defective in purine salvage enzymes. Science. 1977. 197: 1284-1287.

Kamatani N, Carson DA. Dependence of adenine production upon polyamine synthesis in cultured human lymphoblasts. Biochim Biophys Acta. 1981. 675: 344-350.

Kaufmann SH, Desnoyers S, Ottaviano Y, Davidson NE, Poirier GG. Specific proteolytic cleavage of poly (ADP-ribose) polymerase: an early marker of chemotherapy-induced apoptosis. Cancer Res. 1993. 53: 3976-3985.

Kerr JF, Wyllie AH, Currie AR. Apoptosis: a basic biological phenomenon with wide-ranging implications in tissue kinetics. Br J Cancer. 1972. 26: 239-257.

Kishi T, Kittaka E, Hyodo S, Kashiwa H, Karakawa T, Suzawa T, Sakura N, Sakano T, Usui T. Inhibition by adenine of in vitro immunological functions of normal and adenine phosphoribosyltransferase-deficient human lymphocytes. Immuno- pharmacology. 1985. 10: 157-162.

Lai HW, Wei JC, Hung HC, Lin CC. Adenine inhibits the growth of colon cancer cells via AMP-activated protein kinase mediated autophagy. Evid Based Complement Alternat Med. 2019. 2019: 9151070.

Lowe SW, Lin AW. Apoptosis in cancer. Carcinogenesis. 2000. 21: 485-495

Silwal P, Shin K, Choi S, Kang SW, Park JB, Lee HJ, Koo SJ, Chung KH, Namgung U, Lim K, Heo JY, Park JI, Park SK Adenine suppresses IgE-mediated mast cell activation. Mol Immunol. 2015. 65: 242-249.

Simon ER, Chapman RG, Finch CA. Adenine in red cell presservation. J Clin Invest. 1962. 41: 351-359.

Snyder FF, Hershfield MS, Seegmiller JE. Cytotoxic and metabolic effects of adenosine and adenine on human lymphoblasts. Cancer Res. 1978. 38: 2357-2362.

Soengas MS, Lowe SW. Apoptosis and melanoma chemoresistance. Oncogene. 2003. 22: 3138-3151.

Su WW, Huang JY, Chen HM, Lin JT, Kao SH. Adenine inhibits growth of hepatocellular carcinoma cells via AMPK-mediated S phase arrest and apoptotic cascade. Int J Med Sci. 2020. 17: 678-684.

Taylor RC, Cullen SP, Martin SJ. Apoptosis: controlled demolition at the cellular level. Nat Rev Mol Cell Biol. 2008. 9: 231-241.

Theodoropoulou S, Brodowska K, Kayama M, Morizane Y, Miller JW, Gragoudas ES, Vavvas DG. Aminoimidazole carboxamide ribonucleotide (AICAR) inhibits the growth of retinoblastoma in vivo by decreasing angiogenesis and inducing apoptosis. PLoS One. 2013. 8: e52852.

Thornberry NA. The caspase family of cysteine proteases. Br Med Bull. 1997. 53: 478-490.

Vincent EE, Coelho PP, Blagih J, Griss T, Viollet B, Jones RG. Differential effects of AMPK agonists on cell growth and metabolism. Oncogene. 2015. 34: 3627-3639.

Watanabe S, Yoshimi Y, Ikekita M. Neuroprotective effect of adenine on purkinje cell survival in rat cerebellar primary cultures. J Neurosci Res. 2003. 74: 754-759.

Young GH, Lin JT, Cheng YF, Huang CF, Chao CY, Nong JY, Chen PK, Chen HM. Identification of adenine modulating AMPK activation in NIH/3T3 cells by proteomic approach. J Proteomics. 2015. 120: 204-214.

Zadra G, Photopoulos C, Tyekucheva S, Heidari P, Weng QP, Fedele G, Liu H, Scaglia N, Priolo C, Sicinska E, Mahmood U, Signoretti S, Birnberg N, Loda M. A novel direct activator 
of AMPK inhibits prostate cancer growth by blocking lipogenesis. EMBO Mol Med. 2014. 6: 519-538.
https://doi.org/10.15616/BSL.2020.26.3.179

Cite this article as: Silwal P, Park SK. Adenine Inhibits B16-F10 Melanoma Cell Proliferation. Biomedical Science Letters. 2020. 26: 179-185. 